\title{
New observational project for revealing natural and anthropogenic threats at the near-Earth space
}

\author{
H. Harutyunian, E. Nikoghosyan, N. Melikian, N. Azatyan, \\ H. Abrahamyan, G. Paronyan, H. Andreassian, G. Ohanian, M. Gevorgyan, \\ G. Mickaelyan, D. Andreasyan, A. Grigoryan
}

NAS RA V. Ambartsumian Byurakan Astrophysical Observatory (BAO), Armenia

E-mail: hhayk@bao.sci.am

\begin{abstract}
In 2014, a new monitoring project started at the observational base Saravand of the Byurakan astrophysical observatory. This project initiated for revealing natural and artificial objects at the near-Earth space. This is a kind of continuation of earlier observational projects implemented at the observatory prior the collapse of Soviet Union. This time, near-Earth space monitoring is carried out at the request of the Russian agency ROSKOSMOS. For observations, the EOP-1 module is used, which includes small telescopes with a mirror diameter of $40 \mathrm{~cm}, 25 \mathrm{~cm}$ and $19 \mathrm{~cm}$.
\end{abstract}

Keywords: Near-Earth space - artificial satellites - cosmic junk - monitoring

\section{Introduction}

The cosmic epoch started about six decades ago with the launch of Soviet Sputnik 1 in October 4, 1957. During this period, thousands of artificial objects were launched to various orbits, which make the space gradually more and more junked with metallic debris. These are named space junk colloquially. Actually, these are orbital bits that do nothing useful, namely, spent rocket stages, fragments splayed by collisions and erosion, old satellites no one cares about anymore. In total, they amount to millions of pieces of debris, many of which are large enough to damage satellites. Scientists estimate that there are about 7,500 tons of rubbish in space and we are reaching a critical point.The low-Earth orbit is already overpopulated. Even in the unrealistic case of no new launch politics, the number of debris will grow rapidly. The point is that collisions cascade into more collisions, which create more debris that causes more collisions that cascade into more collisions - the effect called Kessler Syndrome.Just one collision in space can create thousands of new highspeed, out-of-control pieces and threaten other spacecraft. 
On the other hand, governmental and private space agencies continue launching new cosmic vehicles increasing the population of unusable garbage in the Earth vicinity. SpaceX alone, for example plans to send up nearly 12,000 small internetbeaming objects over time. Scientists warn that the growing problem of space debris is putting spacecraft and astronauts at risk. It is estimated that there are about half a million pieces of manmade rubbish orbiting the Earth, ranging from huge defunct satellites, to spent rocket boosters, lost astronaut tools and more. This dangerous orbital garbage is moving roughly 10 times faster than a speeding bullet and takes a long time to crash back to earth. These pieces of metal can stay up there very long. For instance, the lifetime of geosynchronous satellites in high orbits can amount even thousands of years.

\section{Early projects at BAO and Armenia for revealing of artificial satellites.}

First observations of artificial satellites in Armenia started in 1957 immediately after the first Soviet Sputnik launch. By the decision of the Astronomical council of the Soviet academy of sciences Yerevan astronomical observatory (Yerevan State University Observatory at present) began observations of the first artificial satellites of the Earth, using the small telescopes for this purpose. The $20 \mathrm{~cm}$ refractor was the largest telescope implemented in this project. Professor Benik Tumanian was the one responsible for observations. There was a small staff implementing observations, and the students of YSU were involved in observations. This project lasted up to beginning of 1970s.

Observations of artificial satellites have become more regular and systematic since 1973, when a department of practical astronomy began its activity in the Byurakan Observatory. That was the time of arms race between "East and West". The number of satellites in circumterrestrial orbits was growing rapidly. In spite the fact that both super states of that time, i.e. the USSR and US were proclaiming their commitment to the ideas of the peaceful usage of the space, some unannounced launches happened periodically. Undoubtedly, the latter ones had military objectives mainly and implemented for reconnaissance data collecting. Potential adversaries used their scientific and technological potential for scouting out the capability of the other side. Therefore, both of the sides continuously looked for any reliable information to contain the assumed enemy. That was the reason for establishing the observational department mentioned above.

Newly organized department was located at Saravand - the area about two km far from the main territory of the BAO, previously used for radio astronomical observations. The staff observed the near-Earth space with several small telescopes, 
mounted under the semi-cylindrical dome. The observers' team, as well as all the engineers providing the maintenance of the equipment have been graduated from Armenian institutes.

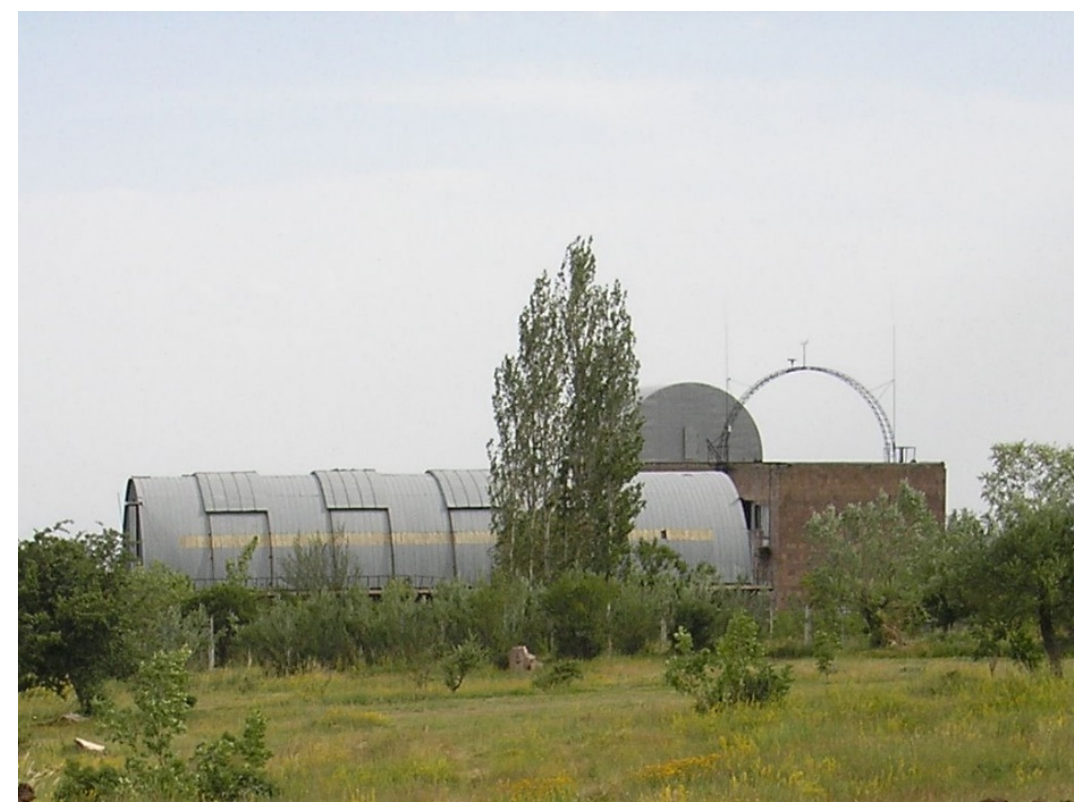

Figure 1. Observational base at the Saravand base, meant for revealing of artificial satellites

The project of revealing the "spy" and lost satellites continued up to the very beginning of 90 s of the last century, right up to collapse of the Soviet Union. After that, all the activity in Byurakan aimed at the monitoring of near-Earth space interrupted for decades. Besides the huge role, which played the collapse of the Soviet Union for this situation, there was another strong factor bringing to stop of the mentioned activity. That was the sharp deterioration in the economic situation of Russia itself, which was the main observer of observational data.

\section{New stage of observations.}

Observations of satellites interrupted when Armenia became sovereign country and old resolutions made by the government of the USSR lost their force. Over time, this followed the devastating earthquake in Armenia, and coincided with military actions on the border of Artsakh with Azerbaijan. The economics of Armenia was completely destroyed, and scientific organizations were surviving at a very low financial level. Interruption of contract made for satellites revealing closed one of serious sources of funding for the Byurakan observatory. Therefore, since 1995 the observatory tried to find ways for restoring the old project or finding new partners to re-operate the unused observational equipment. 


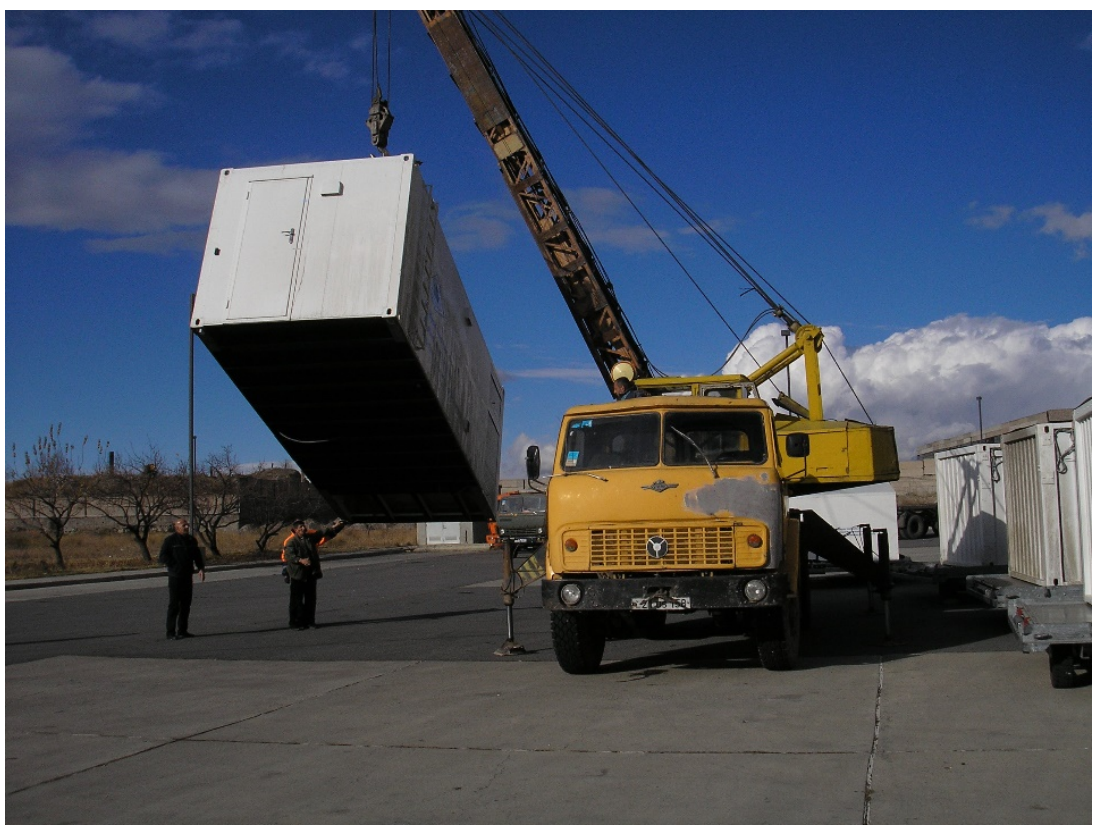

Figure 2. The control room and dome-containers of the module EOP-1 at the airport Zvartnots

However, only about ten years after beginning of activities for recommencement of the mentioned observations appeared some chances for it. The group headed by Igor Molotov from the Keldysh Institute of Applied Mathematics was the first to take note the observational opportunities of the Byurakan observatory potentially qualified for joint research of the near-Earth space. Of course, in the center of their attention were the large telescopes of the BAO. They were interested in using large telescopes of the observatory, since they needed to investigate more closely the near-Earth space in order to detect rather small pieces of cosmic debris. For this purpose, the Russian counterpart even suggested to change optical focuses of the telescopes for providing wider observational field. However, it could mean that the telescopes turn into instruments designed for solving only similar problems, which rejected at the very beginning of cooperation.

Nevertheless, this initiative was continued, and in 2011, the foundation for the observational station EOP-1 was laid at the Saravand base. In parallel, the observatory renewed completely the power supply of the Saravand area, for which the transformer substation was fully equipped. At the end of 2013, the Russian company "Astronomical Scientific Center" airlifted all the necessary equipment of the EOP-1 with small telescopes to Armenian airport Zvartnots. The same day all the equipment with the containers designed as observational domes and control room were transferred to the Saravand area.

In February 2014, the EOP-1 started first observations. Nearly at the same time rebuilding of practically destroyed two-store laboratory building. It turned out to be an incomparably long and hard work, because last almost twenty-five years, passed 
after the last operations in this area, was a period of complete breakdown for this base. During this time, the roof of the building, and then the entire internal structure became into complete disrepair. Repairs in the building are going on up to now, although a huge part is over already.

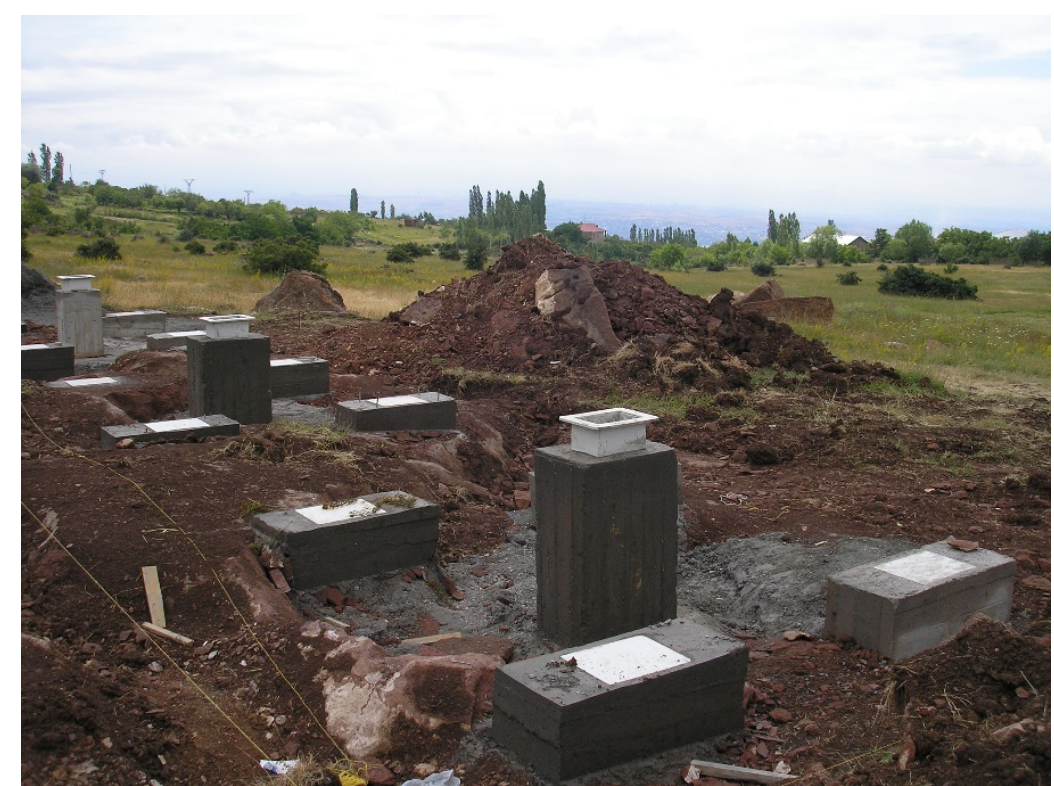

Figure 3. The fundaments of telescopes and containers-domes built at Saravand.

At the beginning of August 2014, the BAO observing crews started observations as well. Since August 2014, four crews have been working each consisting of three observers who perform scheduled observations on a shift basis. Each observation crew consists of two specialists from the Byurakan Observatory and one specialist from Russia. The schedule of work is drawn up in such a way that Armenian participants work every fourth week, and Russian specialists every fourth month. Since the very beginning of these observations, the working conditions improve continuously. Depending on various responsibilities at the observatory as well as on the changes in marital status, some members of crews have left the group of observers and new ones were involved as observers.

\section{EOP-1 module}

It is obvious that for observing the near-Earth space one needs high aperture and wide field telescopes. These telescopes should have focal field of, at least, a few square degrees.

The largest at present telescope operating in the frame of module EOP-1 is the OES-1 with the objective diameter $400 \mathrm{~mm}$ and the focal length $1200 \mathrm{~mm}$. The focal 
field of this telescope has sizes $1.75^{\circ} \times 1.75^{\circ}$ giving total about 3 square degrees. In the dark and astronomically good night, one can reveal objects up to $17.5^{\mathrm{m}}$.

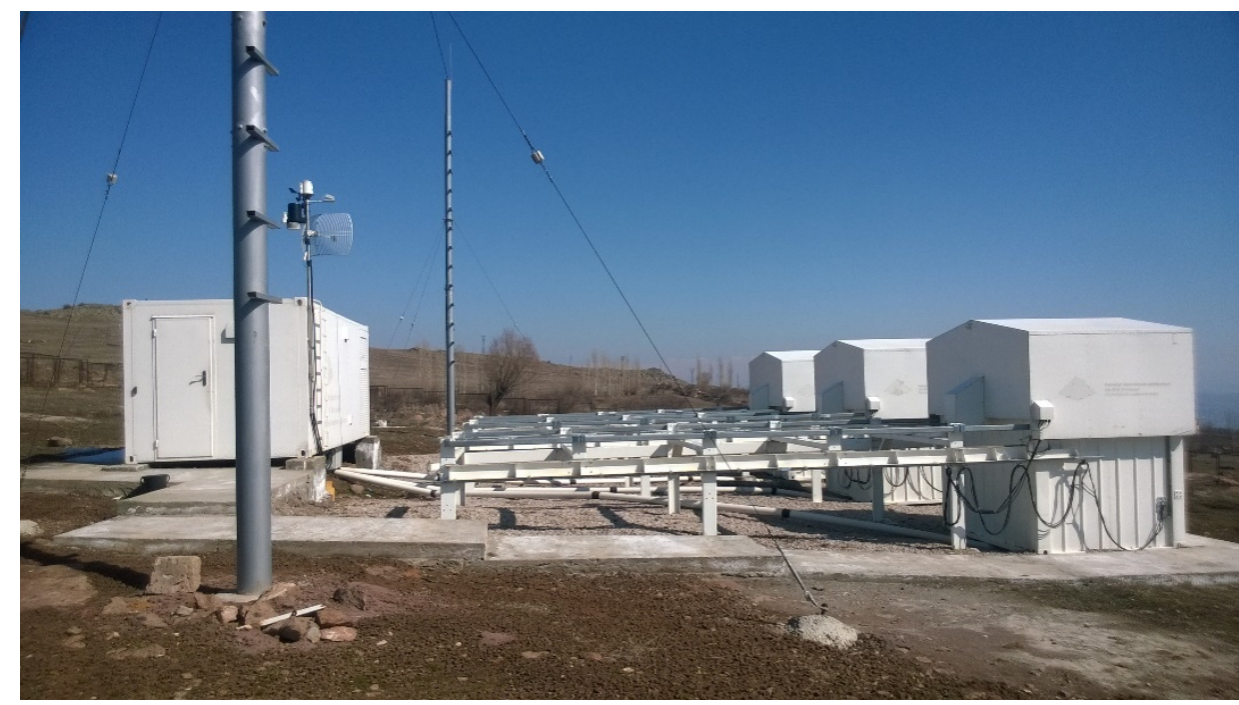

Figure 4. Observational module EOP-1 operating at the Saravand base of the BAO

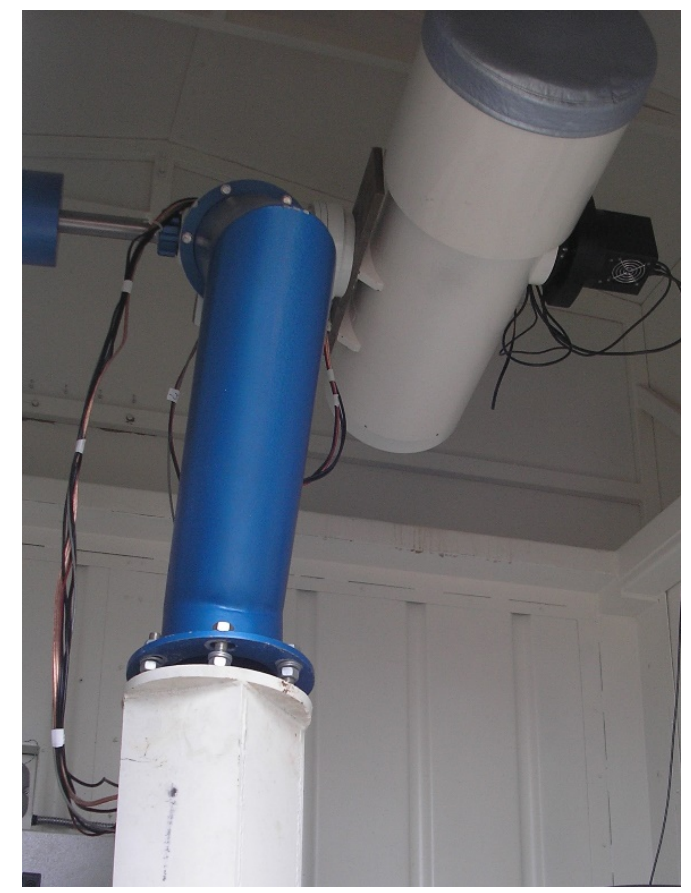

Figure 5. $250 \mathrm{~mm}$ aperture telescope mounted in the container-dome.

The second telescope included into EOP-1 is a little bit smaller, providing good results of observations up to $15.5^{\mathrm{m}}$. This telescope has an aperture, which is equal to $250 \mathrm{~mm}$, and gives rather good images in the rectangular field of sizes $3.3^{\circ} \times 2.2^{\circ}$. The focal length is $627 \mathrm{~mm}$. 
At last, the smallest telescope consists of two identical cameras VT-78 designed by Valeriy Terebizh. Those are $190 \mathrm{~mm}$ aperture and $295 \mathrm{~mm}$ focal length cameras, providing wide rectangular field of sizes $7.1^{\circ} \times 4.7^{\circ}$.

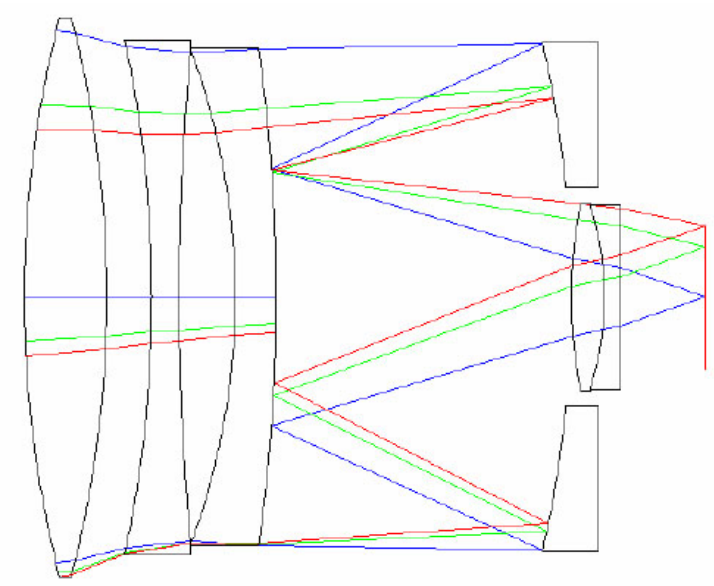

Figure 5. The optical scheme of the camera VT-78.

All these telescopes are equipped with modern light detectors, built on the base of modern CCD matrices. The central computer controlling telescopes guiding and movement collects observational data as well.

\section{Working and living conditions}

The observational base of the BAO at Saravand exists since the beginnings of 1960s. At that time, in this territory there was located the department of radio astronomy of $\mathrm{BAO}$, for the life of which the entire infrastructure was created. There were two laboratory buildings (two-storied and one-storied constructions, built of Byurakan red-yellow tuff), a transformer substation, central heating, a water pipe, etc. In laboratory buildings, all the working rooms, workshops and laboratory facilities were located. When the project aimed to observations of artificial satellites began, a part of the two-storied building was used for the new project, but some new buildings were built as well (some are shown in Fig.1).

As we noticed above the last decade of last century and the first decade, when the Byurakan observatory was in grave conditions, the situation at the Saravand base was much worse. Since these buildings were not used for almost a quarter of a century, all of them were dilapidated, some of them no longer had a roof and the water of atmospheric precipitations flowed to the basement. It was clear that in these conditions, the exploitation of these buildings is impossible. There were few alternatives: either one had to repair the existing buildings, or build a new residential and working module for carrying out the scheduled work on the project. We 
preferred the first alternative, since the existing buildings, although partially fractured due to difficult environments and lack of maintenance, the buildings built about fifty years ago, could provide all the necessary conditions to implement the project. There was a lot of space for offices, bedrooms, a conference hall, a kitchen dining room, domesticity etc. Therefore, the two-storied building was renewed for these purposes.
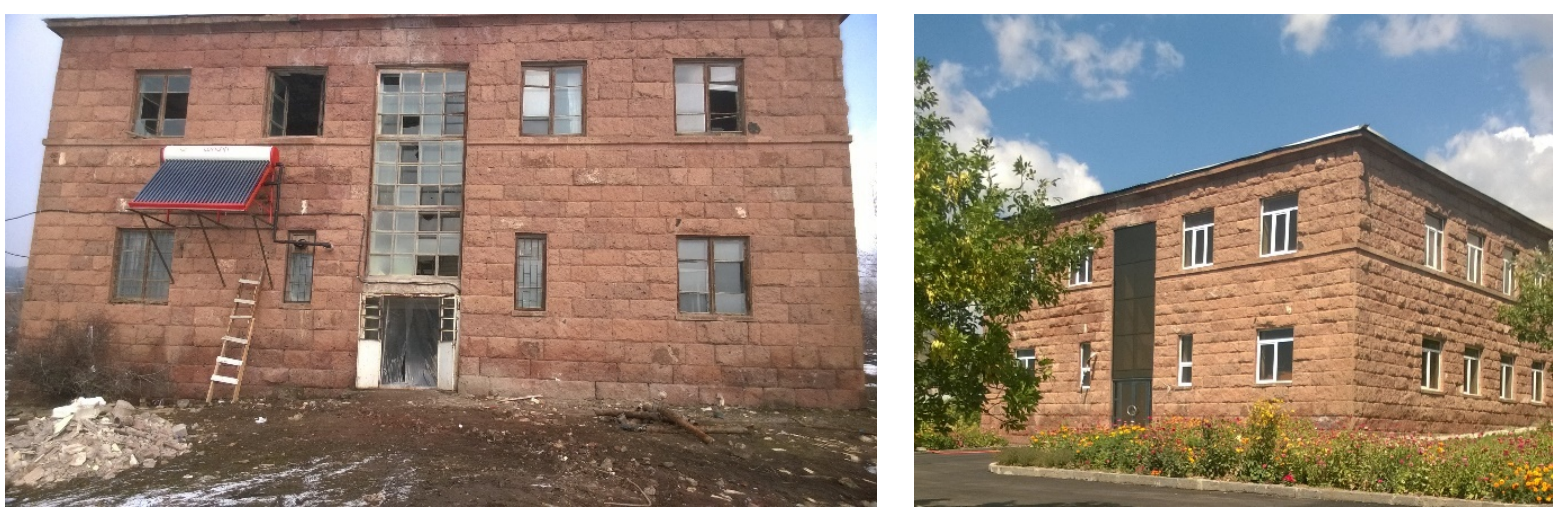

Figure 6. The two-storied laboratory-living building before and after the renewal.

Now, after three years of restoration work, the two-storied laboratory building is almost completely renovated. The renovated building has an autonomous heating system, and observers can stay there and work.

\section{Outlooks}

This project is carried out within the framework of the Center for Applied Astronomy of the Byurakan Observatory. It is envisaged that in the future the monitoring capacities of this station will increase. As a first step, the introduction of a new telescope with an aperture of $650 \mathrm{~mm}$ is being considered. This will make it possible to detect objects at least one stellar magnitude fainter than what is currently possible with a $400 \mathrm{~mm}$ telescope.

On the other hand, the Center for Applied Astronomy undertakes for other activities as well. The Byurakan Observatory and the Corporation "Russian Cosmic Systems" have signed a preliminary Agreement for establishing here also an access point for telecommunication and information exchange. At present, the observatory has only radio connection with the Internet. When this agreement comes into force and the relevant work commences, the Byurakan Observatory will receive a fiberoptic connection to the Internet as a by-product.

Of course, Byurakan astrophysical observatory is established mainly for the fundamental research in the field of astrophysics. Therefore, the Byurakan observatory is well known for its results obtained in the fundamental research, namely, in the fields of stellar and extragalactic astronomy, especially, for the new 
ideas concerning the active phenomena and evolution issues. However, in the modern world adaptation of scientific knowledge to the practical problems becomes more challenging. 